ANNALES

POLONICI MATHEMATICI

$84.2(2004)$

\title{
Translation equation on monoids
}

\author{
by Andrzej Mach (Kielce) and Zenon Moszner (Kraków)
}

\begin{abstract}
We give large classes of solutions of the translation equation on a monoid satisfying the identity condition.
\end{abstract}

Let $X$ be a nonempty set and let $(G, \cdot)$ be a groupoid. By $F: X \times G \rightarrow X$ we denote an arbitrary solution of the translation equation:

$$
F(F(\alpha, k), l)=F(\alpha, k \cdot l), \quad \alpha \in X ; k, l \in G .
$$

This equation appears in several mathematical domains: abstract geometric and algebraic objects, abstract automata, groups of transformations, iterations, representations of groups, dynamical systems and others (see [5]) and therefore has at present a general theory (see [7]).

János Aczél of the University of Waterloo, in a letter to the second author, posed the following problem: what can we say about solutions $F$ : $X \times \mathbb{N} \rightarrow X$ of the translation equation (1) for which $F(\alpha, 1)=\alpha$ (the identity condition), where $X$ is an interval and $(\mathbb{N}, \cdot)$ is the monoid of natural numbers?

We give large classes of solutions of the translation equation on monoids $G$ satisfying the identity condition $F(\alpha, 1)=\alpha$, where 1 denotes the unit element of $(G, \cdot)$.

The problem of finding the general solution of the translation equation for $(G, \cdot)=(\mathbb{N}, \cdot)$ is still open.

REMARK 1 . If $F$ is a solution of (1), then $G$ acts on $X$ by means of the mapping $k \mapsto F(\cdot, k): X \rightarrow X$.

Definition 1. A family $\left\{E_{j}\right\}_{j \in J}$ of nonempty pairwise disjoint subsets of $G$ is called an invariant decomposition of the groupoid $(G, \cdot)$ if $G=\bigcup_{j \in J} E_{j}$ and

$$
\forall j \in J \forall k \in G \exists l \in J: \quad\left(E_{j} \cdot k \subseteq E_{l}\right) .
$$

2000 Mathematics Subject Classification: Primary 39B82; Secondary 39B12, 39B52. Key words and phrases: translation equation, grupoid, monoid, invariant decomposition. 
Theorem 1. Let $X$ be a nonempty set. Let $X=\bigcup_{s \in S} X_{s}$ be a decomposition of $X$ into a disjoint union of nonempty sets such that for every $s \in S$ there exists an invariant decomposition $\left\{E_{j s}\right\}_{j \in J_{s}}$ of the monoid $(G, \cdot)$ with $\operatorname{card} X_{s}=$ card $J_{s}$. Let $\bar{g}_{s}:\left\{E_{j s}\right\}_{j \in J_{s}} \rightarrow X_{s}$ be an arbitrary bijection and set $g_{s}(k):=\bar{g}_{s}\left(E_{j s}\right)$ for $k \in E_{j s}$. Then the function $F: X \times G \rightarrow X$ defined by

$$
F(\alpha, k)=\} g_{s}\left(g_{s}^{-1}(\{\alpha\}) \cdot k\right)\left\{, \quad \alpha \in X_{s}, k \in G,\right.
$$

is a solution of the translation equation (1) for which $F(\alpha, 1)=\alpha$.

The symbol $\} A\{$ in (3) denotes the element of a set $A$ when card $A=1$.

The proof of Theorem 1 is a simple verification, so it can be omitted.

REMARK 2. The decomposition $\left\{E_{j}\right\}_{j \in J}$ of $G$ is invariant if and only if the relation

$$
a \equiv b \Leftrightarrow \exists j \in J: a, b \in E_{j}
$$

is right-compatible with the groupoid operation, i.e.

$$
\forall a, b, c \in G: \quad[a \equiv b \Rightarrow a \cdot c \equiv b \cdot c] .
$$

If the groupoid $G$ is Abelian, then every equivalence relation $\equiv$ rightcompatible with the groupoid operation is a congruence relation, that is,

$$
\forall a, b, c, d \in G: \quad[(a \equiv b \wedge c \equiv d) \Rightarrow a \cdot c \equiv b \cdot d] .
$$

An equivalence relation $\equiv$ on a groupoid $G$ is a congruence (respectively: is right-compatible with the groupoid operation) if and only if there exists a function $h: G \rightarrow G$ such that

$$
a \equiv b \Leftrightarrow h(a)=h(b) \text { and } h(a \cdot b)=h[h(a) \cdot h(b)]
$$

(respectively: $h(a \cdot b)=h[h(a) \cdot b]$ ) for $a, b \in G$.

In the case of a congruence relation, the function $h$ is a homomorphism of $G$ onto the groupoid $h(G)$ with the operation $c \# d=h(c \cdot d)$. This means that the equivalence relation $\equiv$ is a congruence in the groupoid $(G, \cdot)$ if and only if there exists a homomorphism $H$ of $G$ into a groupoid $T$ such that $a \equiv b \Leftrightarrow H(a)=H(b)$ (see [2, pp. 35-37]). This yields a method of constructing invariant decompositions (see Remark 4, due to Andrzej Schinzel).

REMARK 3. If the groupoid $G$ is a group, then its invariant decompositions are sets of right cosets of some subgroup (see [1, pp. 34-35]). Moreover, if the group $G$ is Abelian then invariant decompositions are determined by quotient groups.

Remark 4 (by A. Schinzel). By Remark 2 all congruences $\equiv$ in the monoid $(\mathbb{N}, \cdot)$ are obtained by the following 


\section{Construction $C_{1}$}

$1^{\circ}$ Take an arbitrary Abelian semigroup $(T,+)$ with neutral element 0.

$2^{\circ}$ Take an arbitrary function $\phi: P \rightarrow T$, where $P$ is the set of all prime numbers. Define a homomorphism $H:(\mathbb{N}, \cdot) \rightarrow(T,+)$ by setting, for $a=\prod_{p \in P} p^{\alpha(p)} \in \mathbb{N}$, where $\alpha(p)$ are nonnegative integers,

$$
H(a):=\sum_{p \in P} \alpha(p) \phi(p)
$$

$3^{\circ}$ For $a, b \in \mathbb{N}$ define: $a \equiv b \Leftrightarrow H(a)=H(b)$, that is,

$$
\prod_{p \in P} p^{\alpha(p)} \equiv \prod_{p \in P} p^{\beta(p)} \Leftrightarrow \sum_{p \in P} \alpha(p) \phi(p)=\sum_{p \in P} \beta(p) \phi(p),
$$

where $\alpha(p), \beta(p)$ are nonnegative integers.

To describe all congruence relations means to describe all semigroups and, in consequence, to solve the association equation

$$
F(F(a, b), c)=F(a, F(b, c)), \quad \text { where } \quad F: G \times G \rightarrow G .
$$

EXAmple 1 (by A. Schinzel). Let $T:=2^{\mathbb{N}}$ be the monoid with the union operation. If we define $\phi(p):=\{p\}$ we get the congruence relation

$$
a \equiv b \Leftrightarrow a \text { and } b \text { have the same prime factors; }
$$

this means that components of the invariant decomposition of $\mathbb{N}$ are sets of natural numbers having the same prime factors.

REMARK 5. To obtain the same invariant decomposition as in Example 1, it is possible to take $\mathbb{N}$ with a suitable operation in place of $2^{\mathbb{N}}$. The function $h: \mathbb{N} \rightarrow \mathbb{N}$ such that $h(a)$ equals the product of the prime factors of $a$ for $a>1$ and $h(1)=1$ satisfies (4), hence $h$ is a homomorphism of $(\mathbb{N}, \cdot)$ into $\mathbb{N}$ with the operation $a \# b=h(a \cdot b)$.

REMARK 6 . When the monoid $(G, \cdot)$ is the group then Theorem 1 yields all solutions of the translation equation (1) satisfying $F(\alpha, 1)=\alpha$. In this case the invariant decompositions consist of right cosets of some subgroup $G_{s}$ of $G$ (see Remark 3 ) and $\bar{g}_{s}$ is equal to $g_{s}$ and $g_{s}: G / G_{s} \rightarrow X_{s}$. Also the general solution of the translation equation (1) satisfying $F(\alpha, 1)=\alpha$ has been given in [4] by the following

\section{Construction $C_{2}$}

$1^{\circ}$ Let $X=\bigcup_{s \in S} X_{s}$ be a disjoint union of nonempty sets (fibres) $X_{s}$ such that for every $s \in S$ there exists a subgroup $G_{s} \leq G$ and a bijection $g_{s}: G / G_{s} \rightarrow X_{s}$, where $G / G_{s}$ is the set of right cosets of $G_{s}$ in $G$.

$2^{\mathrm{o}}$ Then $F(\alpha, k)=g_{s}\left(g_{s}^{-1}(\alpha) \cdot k\right), \alpha \in X_{s}, k \in G$. 
Remark 7. Construction $C_{3}$ mentioned below, quoted from [3], includes the general form of invariant decompositions for the subsemigroup $G^{+}$of positive elements of the group $(G,+, \leq)$, linearly ordered and Abelian.

Before the presentation of the construction of the decompositions, we need two definitions.

Definition 2. A subset $A$ of $G$ is called bounded if $\exists z \in G^{+} \forall a \in A$ : ( $a<z$ and $a>-z$ ), and unbounded if it is not bounded.

Definition 3. Let $A, B$ be subsets of $G$ and $A \subseteq B \subseteq G$. We say that:

(a) $A$ is an initial interval of $B$ if

$$
\forall a_{0} \in A: \quad\left\{a \in B: a \leq a_{0}\right\} \subseteq A,
$$

(b) $A$ is a final interval of $B$ if

$$
\forall a_{0} \in A: \quad\left\{a \in B: a_{0} \leq a\right\} \subseteq A .
$$

All $G^{+}$-invariant decompositions of the semigroup $G^{+}$of positive elements of a linearly ordered, Abelian group $G$ are obtained by

Construction $C_{3}$

$1^{\mathrm{O}}$ Take a family $\left\{G_{s}\right\}_{s \in S}$ of distinct, bounded subgroups of $G$ forming a chain, i.e. $G_{s} \subset G_{t}$ or $G_{t} \subset G_{s}$ for $s, t \in S$, and an unbounded subgroup $G^{*}$ such that $G^{*} \supseteq \bigcup_{s \in S} G_{s}$.

$2^{\circ}$ Let $\Phi$ be a function from the family $\left\{G_{s}\right\}_{s \in S}$ onto a family of initial intervals of $G^{+}$such that

(a) $\Phi\left(G_{s}\right)$ is a union of intersections with $G^{+}$of cosets of $C\left(G_{s}\right)$ in $G$, where $C\left(G_{s}\right)$ denotes the smallest convex subgroup containing $G_{s}$ (the convexity of $C\left(G_{s}\right)$ means that together with every positive element a the subgroup $C\left(G_{s}\right)$ contains all elements $x \in G^{+}$with $x \leq a)$,

(b) if $G_{s} \subset G_{t}$, then $\Phi\left(G_{s}\right) \subset \Phi\left(G_{t}\right)$.

$3^{\circ}$ Every nonempty set

$$
W \cap\left[\Phi\left(G_{s}\right) \backslash \bigcup_{G_{t} \varsubsetneqq G_{s}} \Phi\left(G_{t}\right)\right], \quad W \in G_{s}, s \in S,
$$

is a component of the decomposition.

$4^{\circ}$ The sets

$$
V \cap\left[G^{+} \backslash \bigcup_{s \in S} \Phi\left(G_{s}\right)\right], \quad V \in G / G^{*},
$$

are the remaining components.

If we assume additionally that $(G,+, \leq)$ is an Archimedian group, then Construction $C_{3}$ is reduced to the following result from the paper [6]. 
Every $G^{+}$-invariant decomposition of the semigroup $G^{+}$of positive elements of a linearly ordered, Archimedian group is of the following form:

(a) there exists a right-closed or right-open interval $\left[0, x_{0} \mid\right.$ such that every element belonging to $\left[0, x_{0} \mid\right.$ is a component of the decomposition (this interval may be empty),

(b) the remaining components are the intersections with $G^{+} \backslash\left[0, x_{0} \mid\right.$ of cosets of some subgroup $G^{*}$ in $G$.

Using Construction $C_{3}$ and Theorem 1 we can obtain examples of solutions for the semigroup $G^{+}$of positive elements of a linearly ordered, Abelian group $(G,+, \leq)$.

EXAMPLE 2. Let $\mathbb{Z}$ denote the set of integes and $G:=\{a x+b: a, b \in \mathbb{Z}\}$ be the group of linear polynomials with ordinary addition and with linear order defined as follows:

$$
(a x+b \leq c x+d) \Leftrightarrow(a<c) \text { or }(a=c \text { and } b \leq d) .
$$

The semigroup of positive elements is

$$
G^{+}:=\{a x+b: a>0, b \in \mathbb{Z}\} \cup \mathbb{Z}^{+},
$$

where $\mathbb{Z}^{+}:=\{a \in \mathbb{Z}: a \geq 0\}$. According to Construction $C_{3}$, take the chain of bounded subgroups $\{0\} \subset \mathbb{Z}$ and the unbounded subgroup $G^{*}:=G$. Define $\Phi(\{0\}):=\mathbb{Z}^{+}$and $\Phi(\mathbb{Z}):=\mathbb{Z}^{+} \cup(\mathbb{Z}+x)$, where $\mathbb{Z}+x \in G / \mathbb{Z}$. Every element of $\mathbb{Z}^{+}$is a component of the decomposition. The sets $\mathbb{Z}+x$, $G^{+} \backslash\left(\mathbb{Z}^{+} \cup(\mathbb{Z}+x)\right)$ are also components.

Let now $X:=\left[0, \infty\left[, S:=\left[0,1\left[, X_{s}:=\{s+j: j=0,1,2, \ldots\}\right.\right.\right.\right.$ and $J_{s}:=\mathbb{N} \cup\{0\}$ for $s \in S$. Moreover, $E_{0 s}:=\mathbb{Z}+x, E_{1 s}:=G^{+} \backslash\left(\mathbb{Z}^{+} \cup(\mathbb{Z}+x)\right)$ and $E_{j s}:=\{j-2\}$ for $j \in\{2,3,4, \ldots\}$ and $s \in S$. Assume that $\bar{g}_{s}\left(E_{j s}\right):=s+j$ for $j \in \mathbb{N} \cup\{0\}$ and for $s \in S$. We get the following solution $F: X \times G^{+} \rightarrow X$ of the translation equation:

$$
F(\alpha, w)= \begin{cases}\alpha & \text { if } \alpha \in\left[0,1\left[\text { and } w \in \mathbb { Z } ^ { + } \text { or } \alpha \in \left[1,2\left[\text { and } w \in G^{+},\right.\right.\right.\right. \\ \alpha+1 & \text { if } \alpha \in\left[0,1\left[\text { and } w \in G^{+} \backslash \mathbb{Z}^{+},\right.\right. \\ \alpha-E(\alpha) & \text { if } \alpha \in X \backslash[0,2[\text { and } w \in \mathbb{Z}+x, \\ \alpha-E(\alpha)+1 & \text { if } \alpha \in X \backslash\left[0,2\left[\text { and } w \in G^{+} \backslash\left(\mathbb{Z}^{+} \cup(\mathbb{Z}+x)\right),\right.\right. \\ \alpha+w & \text { if } \alpha \in X \backslash\left[0,2\left[\text { and } w \in \mathbb{Z}^{+},\right.\right.\end{cases}
$$

where $E(\alpha)$ denotes the integer part of $\alpha$.

In what follows $(\mathbb{N}, \cdot)$ and $\left(\mathbb{Q}_{+}, \cdot\right)$ denote the monoid of natural numbers and the group of positive rational numbers respectively.

Using Theorem 1 we can obtain examples of solutions for $(G, \cdot)=(\mathbb{N}, \cdot)$.

ExAmple 3. Let $X:=] 1 / 4,1]$ and take $\left.\left.S:=] 1 / 2,1], X_{s}:=\right] s / 2, s\right]$, $J_{s}:=\{1,2\}$ for $s \in S$. Moreover, $E_{1 s}:=\{1,3,5, \ldots\}, E_{2 s}:=\{2,4,6, \ldots\}$ for 
$s \in S$. Define $\bar{g}_{s}\left(E_{1 s}\right):=s / 2, \bar{g}_{s}\left(E_{2 s}\right):=s$ for $s \in S$. We get the following solution:

$$
F(\alpha, k)= \begin{cases}2 \alpha & \text { for } \alpha \in] 1 / 4,1 / 2], k \in\{2,4,6, \ldots\}, \\ \alpha & \text { for } \alpha \in] 1 / 2,1], k \in \mathbb{N} \text { or } \alpha \in] 1 / 4,1 / 2], k \in\{1,3,5, \ldots\} .\end{cases}
$$

Example 4. Let $X, S,\left\{X_{s}\right\}, J_{s}$ for $s \in S$ be as in Example 3. We take $E_{1 s}:=\{1\}, E_{2 s}:=\mathbb{N} \backslash\{1\}$ for $s \in S$. The functions $\bar{g}_{s}$ are defined as in Example 2. We get the following solution:

$$
F(\alpha, k)= \begin{cases}2 \alpha & \text { for } \alpha \in] 1 / 4,1 / 2], k \in \mathbb{N} \backslash\{1\}, \\ \alpha & \text { for } \alpha \in] 1 / 2,1], k \in \mathbb{N} \text { or } \alpha \in] 1 / 4,1 / 2], k=1 .\end{cases}
$$

Example 5. Let $X:=\left[0, \infty\left[, S:=\left[0,1\left[, X_{s}:=\{s+j: j=0,1,2, \ldots\}\right.\right.\right.\right.$, $J_{s}:=\mathbb{N} \cup\{0\}$ for $s \in S$. Moreover, $E_{0 s}:=\{2,4,6, \ldots\}$ and $E_{j s}:=\{2 j-1\}$ for $j \in \mathbb{N}$ and $s \in S$. Define $\bar{g}_{s}\left(E_{j s}\right):=s+j$ for $j \in \mathbb{N} \cup\{0\}$ and $s \in S$. We get the following solution:

$$
\begin{aligned}
& F(\alpha, k) \\
& =\left\{\begin{array}{rr}
\alpha-E(\alpha) & \text { for } \alpha \in X \backslash[0,1[\text { and } k \in\{2,4,6, \ldots\}, \\
& \text { or } \alpha \in[0,1[\text { and } k \in \mathbb{N}, \\
\alpha+E(\alpha)(k-1)-(k-1) / 2 & \text { for } \alpha \in X \backslash[0,1[\text { and } k \in\{1,3,5, \ldots\},
\end{array}\right.
\end{aligned}
$$

where $E(\alpha)$ denotes the integer part of $\alpha$.

Remark 8. If we define $\phi: P \rightarrow T=2^{\mathbb{N}}$ by $\phi(p):=\emptyset$ for $p \neq 2$ and $\phi(2):=\{1\}$, where $T=2^{\mathbb{N}}$ denotes the monoid described in Example 1, then by Construction $C_{1}\left(3^{\circ}\right)$ in Remark 4 we get the congruence equivalent to the invariant decomposition from Example 3, which means that $E_{1}:=$ $\{1,3,5, \ldots\}, E_{2}:=\{2,4,6, \ldots\}$.

Similarly, if we define $\phi: P \rightarrow T=2^{\mathbb{N}}$ by $\phi(p):=\mathbb{N}$ for all $p \in P$, then by Construction $C_{1}\left(3^{\circ}\right)$ we get the congruence equivalent to the invariant decomposition from Example 4, which means that $E_{1}:=\{1\}, E_{2}:=\mathbb{N} \backslash\{1\}$.

To obtain the invariant decomposition from Example 5, it is sufficient to consider the semigroup $(T, \cdot):=\left(2^{\mathbb{R} \backslash\{0\}}, \cdot\right)$, where the operation is defined by $A \cdot B:=\{a \cdot b: a \in A, b \in B\}$ for $A, B \in 2^{\mathbb{R} \backslash\{0\}}$, and to define $\phi: P \rightarrow$ $T=2^{\mathbb{R} \backslash\{0\}}$ by $\phi(p):=\{p\}$ for $p \neq 2$ and $\phi(2):=\mathbb{R} \backslash\{0\}$.

REMARK 9. If the solution of equation (1) is trivial, that is, $F(\alpha, k):=\alpha$ for every $(\alpha, k) \in X \times \mathbb{N}$, where $X$ denotes an arbitrary nonempty set, then the invariant decomposition of $\mathbb{N}$ has exactly one element $\{\mathbb{N}\}$, the set $X$ is decomposed into singletons and $\bar{g}_{s}(\mathbb{N}):=s$.

Remark 10. The function $F(\alpha, k):=k \cdot \alpha$ for $(\alpha, k) \in X \times \mathbb{N}$ and $X:=] 0, \infty[$ is a solution of the translation equation (1). This solution is not of the form (3) (see Remark 11). 
TheOREm 2. Let $X \subset \mathbb{R}$ be an arbitrary interval. Suppose that a solution $F: X \times \mathbb{N} \rightarrow X$ of the translation equation (1) satisfying $F(\alpha, 1)=\alpha$ for $\alpha \in X$ can be extended to a solution $\bar{F}: X \times \mathbb{Q}_{+} \rightarrow X$ of this equation. Then there exists a family $\left\{X_{s}\right\}_{s \in S}$ of disjoint sets such that $\bigcup_{s \in S} X_{s}=X$ and for every $s \in S$ there exists a subgroup $\mathbb{Q}_{s} \leq \mathbb{Q}_{+}$and a bijection $g_{s}$ : $\mathbb{Q}_{+} / \mathbb{Q}_{s} \rightarrow X_{s}$ for which

$$
F(\alpha, k)=g_{s}\left(g_{s}^{-1}(\alpha) \cdot k\right), \quad \alpha \in X_{s}, k \in \mathbb{N} .
$$

Proof. This follows immediately from Construction $C_{2}$.

Theorem 3. Let $X \subset \mathbb{R}$ be an arbitrary interval. A function $F: X \times$ $\mathbb{N} \rightarrow X$ is a solution of the translation equation (1) such that for every $\alpha \in X$ the function $F(\alpha, \cdot)$ is increasing and for every $k \in \mathbb{N}$ the function $F(\cdot, k)$ is increasing and surjective if and only if there exists a family $\left\{X_{s}\right\}_{s \in S}$ of disjoint sets such that $\bigcup_{s \in S} X_{s}=X$ and there exists a family of increasing bijections $g_{s}: \mathbb{Q}_{+} \rightarrow X_{s}, s \in S$, such that

$$
F(\alpha, k)=g_{s}\left(g_{s}^{-1}(\alpha) \cdot k\right), \quad \alpha \in X_{s}, k \in \mathbb{N} .
$$

We present two proofs of this theorem. The first one is a corollary from Theorem 2 and the other proof is direct.

Proof I (of the "only if" part of Theorem 3, using Theorem 2). Note that the assumptions about $F: X \times \mathbb{N} \rightarrow X$ imply that $F(\alpha, 1)=\alpha$ and $F$ can be extended to a solution $\bar{F}: X \times \mathbb{Q}_{+} \rightarrow X$ of (1). Indeed, since $F(F(\alpha, 1), 1)=F(\alpha, 1)$, by injectivity of $F(\cdot, 1)$ we get $F(\alpha, 1)=\alpha$. We can put

$$
\bar{F}(\alpha, k / l):=\beta \quad \text { such that } \quad F(\alpha, k)=F(\beta, l),
$$

for every $\alpha \in X$ and $k / l \in \mathbb{Q}_{+}$. The existence and uniqueness of $\beta$ result from the assumption that $F(\cdot, l)$ is surjective and injective, so $\bar{F}$ is correctly defined. One can verify easily that $\bar{F}$ is a solution of the translation equation. Indeed, let

$$
\bar{F}\left(\bar{F}\left(\alpha, \frac{k}{l}\right), \frac{m}{n}\right)=: \gamma \quad \text { and } \quad \bar{F}\left(\alpha, \frac{k \cdot m}{l \cdot n}\right)=: \delta .
$$

If we set $\bar{F}(\alpha, k / l)=$ : $\beta$, then by definition $(7), F(\alpha, k)=F(\beta, l)$ and $F(\beta, m)=F(\gamma, n)$ and $F(\alpha, k \cdot m)=F(\delta, l \cdot n)$. Hence

$$
F(\gamma, n \cdot l)=F(\beta, m \cdot l)=F(\alpha, k \cdot m)=F(\delta, n \cdot l),
$$

so $\gamma=\delta$.

Therefore, by Theorem 2, we have the form (5) of the solution $F$ : $X \times \mathbb{N} \rightarrow X$. Since the functions $F(\alpha, \cdot)$ are injective for every $\alpha \in X$, by Construction $C_{2}$ we get $\mathbb{Q}_{s}=\{1\} \leq \mathbb{Q}_{+}$for every $s \in S$, which yields (6). 
We will verify that the bijections $\left\{g_{s}\right\}_{s \in S}$ are increasing. Let $k / l<k_{1} / l_{1}$ and

$$
g_{s}(k / l)=: \alpha, \quad g_{s}\left(k_{1} / l_{1}\right)=: \beta .
$$

By (6) and by definition of $\bar{F}$ we get $\bar{F}\left(\alpha, \frac{k_{1} l}{k l_{1}}\right)=g_{s}\left(\frac{k}{l} \cdot \frac{k_{1} l}{k l_{1}}\right)=g_{s}\left(\frac{k_{1}}{l_{1}}\right)=\beta, \quad$ whence $\quad F\left(\alpha, k_{1} l\right)=F\left(\beta, k l_{1}\right)$.

Since $k l_{1}<l k_{1}$, by assumptions we have

$$
F\left(\alpha, k l_{1}\right)<F\left(\alpha, k_{1} l\right)=F\left(\beta, k l_{1}\right) \quad \text { and } \quad \alpha<\beta .
$$

Since the "if" part is evident, the first proof is complete.

Proof II (of the "only if" part of Theorem 3). We define the following relation in $X$ :

$$
\forall \alpha, \beta \in X: \quad \alpha \sim_{F} \beta \Leftrightarrow \exists k, l \in \mathbb{N}: F(\alpha, k)=F(\beta, l) .
$$

It is to verify that it is an equivalence relation. Indeed, evidently it is symmetric and reflexive. Let now $\alpha \sim_{F} \beta$ and $\beta \sim_{F} \gamma$. Then

$$
\exists k, l, k_{1}, l_{1} \in \mathbb{N}: \quad F(\alpha, k)=F(\beta, l) \text { and } F\left(\beta, k_{1}\right)=F\left(\gamma, l_{1}\right) .
$$

Hence

$$
F\left(\alpha, k \cdot k_{1}\right)=F\left(\beta, l \cdot k_{1}\right)=F\left(\gamma, l \cdot l_{1}\right), \quad \text { so } \quad \alpha \sim_{F} \gamma
$$

We denote by $\left\{X_{s}\right\}_{s \in S}$ the set of equivalence classes. Fix $s \in S$ and $\alpha_{0} \in X_{s}$. We define $h_{s}: X_{s} \rightarrow \mathbb{Q}_{+}$by

$$
h_{s}(\alpha):=k / l, \quad \text { where } F\left(\alpha_{0}, k\right)=F(\alpha, l) .
$$

The function $h_{s}$ is correctly defined. Indeed, if

$$
F\left(\alpha_{0}, k\right)=F(\alpha, l) \text { and } F\left(\alpha_{0}, k_{1}\right)=F\left(\alpha, l_{1}\right),
$$

then

$$
F\left(\alpha_{0}, l \cdot k_{1}\right)=F\left(\alpha, l \cdot l_{1}\right)=F\left(\alpha_{0}, l_{1} \cdot k\right) .
$$

Since $F\left(\alpha_{0}, \cdot\right)$ is injective, $l \cdot k_{1}=l_{1} \cdot k$, whence $k_{1} / l_{1}=k / l$.

We will show that $h_{s}: X_{s} \rightarrow \mathbb{Q}_{+}$is a bijection. If $h_{s}(\alpha)=h_{s}(\beta)=$ $k / l$ then $F(\alpha, l)=F\left(\alpha_{0}, k\right)=F(\beta, l)$ and by injectivity of $F(\cdot, l)$ we get $\alpha=\beta$. To prove the surjectivity, take $m / n \in \mathbb{Q}_{+}$. Let $F\left(\alpha_{0}, m\right)=\beta$. By the surjectivity of $F(\cdot, n)$, we have $F\left(\alpha_{0}, m\right)=\beta=F(\alpha, n)$ for some $\alpha$, so $h_{s}(\alpha)=m / n$.

Now, we will show that $h_{s}$ is an increasing function. Let $\alpha<\beta$ and

$$
h_{s}(\alpha)=k / l, \quad h_{s}(\beta)=k_{1} / l_{1} .
$$

We have $F\left(\alpha_{0}, k\right)=F(\alpha, l)$ and $F\left(\alpha_{0}, k_{1}\right)=F\left(\beta, l_{1}\right)$. Since $F\left(\cdot, l l_{1}\right)$ is increasing, we obtain

$$
F\left(\alpha_{0}, k l_{1}\right)=F\left(\alpha, l l_{1}\right)<F\left(\beta, l l_{1}\right)=F\left(\alpha_{0}, k_{1} l\right),
$$

therefore $k l_{1}<k_{1} l$ and $k / l<k_{1} / l_{1}$. 
Let now $\alpha \in X_{s}, k \in \mathbb{N}$. Let $h_{s}(\alpha)=K / L$ and $\beta:=F(\alpha, k)$. Hence

$$
h_{s}(\alpha) \cdot k=K \cdot k / L \text {. }
$$

We will show

$$
h_{s}(\beta)=K \cdot k / L \text {. }
$$

Indeed, we have $F(\beta, L)=F(\alpha, k L), F\left(\alpha_{0}, K\right)=F(\alpha, L)$ and

$$
F\left(\alpha_{0}, K k\right)=F(\alpha, L k)=F(\beta, L),
$$

therefore $h_{s}(\beta)=K \cdot k / L$, and so

$$
F(\alpha, k)=\beta=h_{s}^{-1}(K \cdot k / L)=h_{s}^{-1}\left(h_{s}(\alpha) \cdot k\right) .
$$

Putting $g_{s}=h_{s}^{-1}$ we have the form (6), which was to be shown.

REMARK 11. If $F: X \times \mathbb{N} \rightarrow X$ satisfies the assumptions of Theorem 3, then $F$ cannot be obtained by means of Theorem 1 .

Indeed, otherwise let $g_{s}(1)=: \alpha_{0}$ for some $s \in S$. Then $X_{s}=\left\{F\left(\alpha_{0}, k\right)\right.$ : $k \in \mathbb{N}\}=g_{s}(\mathbb{N})$ and $\alpha_{0} \in X_{s}$. Let $\bar{F}: X \times \mathbb{Q}_{+} \rightarrow X$ be an extension of the solution $F$. Since $\bar{F}\left(\alpha_{0}, 1 / 2\right)<F\left(\alpha_{0}, k\right)$ for $k \in \mathbb{N}$, we have $\bar{F}\left(\alpha_{0}, 1 / 2\right) \notin X_{s}$. Let $\bar{F}\left(\alpha_{0}, 1 / 2\right) \in X_{t}, t \neq s$. Hence

$$
F\left(\bar{F}\left(\alpha_{0}, 1 / 2\right), 2\right)=F\left(\alpha_{0}, 1\right)=\alpha_{0},
$$

so $\alpha_{0} \in X_{t}$, which contradicts the relation $X_{t} \cap X_{s}=\emptyset$.

Remark 12. Let $X:=[0, \infty[$ and define $F: X \times \mathbb{N} \rightarrow X$ by

$$
F(\alpha, k)= \begin{cases}\alpha, & \alpha \in X, k=1, \\ 1, & \alpha \in[0,1], k \in \mathbb{N} \backslash\{1\}, \\ k \alpha, & \alpha \in X \backslash[0,1], k \in \mathbb{N} \backslash\{1\} .\end{cases}
$$

Then $F$ is a solution of (1) which cannot be extended to a solution $\bar{F}$ : $X \times \mathbb{Q}_{+} \rightarrow X$ and is not of the form (3).

Indeed, for every solution $\bar{F}: X \times \mathbb{Q}_{+} \rightarrow X$ of (1) satisfying $\bar{F}(\alpha, 1)=\alpha$, all functions $\bar{F}(\cdot, k)$ ought to be bijections. But

$$
F(1 / 2,2)=1=F(3 / 4,2),
$$

therefore $F$ cannot be extended to a solution $\bar{F}: X \times \mathbb{Q}_{+} \rightarrow X$.

Moreover, by Theorem $1, \operatorname{card} X_{s}=\operatorname{card} J_{s}$ for $s \in S$. It is easy to see that for the solution $F$ one of the elements of the family $\left\{X_{s}\right\}_{s \in S}$ is the set $X_{n}=[0,1]$ for some $n \in S$. This implies the following contradiction:

$$
\mathfrak{c}=\operatorname{card}[0,1]=\operatorname{card} J_{n} \leq \operatorname{card} \mathbb{N}=\aleph_{0} .
$$

\section{References}

[1] N. Bourbaki, Elements of Mathematics, Algebra I, Chapters 1-3, Springer, New York, 1989. 
[2] G. Grätzer, Universal Algebra, Springer, New York, 1968.

[3] A. Mach, Sur les décompositions invariants du demi-groupe du groupe abélien linéairement ordonné, Rocznik Nauk.-Dydak. WSP w Krakowie, Prace Matematyczne 11 (1985), 119-145.

[4] Z. Moszner, Structures de l'automate plein, réduit et inversible, Aequationes Math. 9 (1973), 46-59.

[5] - The translation equation and its application, Demonstratio Math. 6 (1973), 309-327.

[6] -, Décompositions invariants du demi-groupe des éléments non-négatifs du groupe archimédien, Tensor (N.S.) 34 (1980), 8-10.

[7] —, General theory of the translation equation, Aequationes Math. 50 (1995), 17-37.

Institute of Mathematics

Świętokrzyska Academy

Świętokrzyska 15

25-406 Kielce, Poland

E-mail: amach@pu.kielce.pl
Institute of Mathematics Pedagogical Academy Pochorążych 2 30-084 Kraków, Poland E-mail: zmoszner@ap.krakow.pl

Reçu par la Rédaction le 11.3.2004

Révisé le 21.10.2004 